\section{Staging, treatment protocols and outcome in cats with sinonasal carcinomas treated with radiotherapy: a single centre retrospective study of 16 cases}

\section{Petros S Frezoulis, Aaron Harper, Sarah L Mason}

Southfields Veterinary Specialists, Laindon, Essex, United Kingdom

\section{OBJECTIVES}

The radiation treatment ( $R T$ ) protocols and disease outcome in cats with sinonasal carcinomas are poorly described in the literature. This study aimed to describe the outcome of cats following RT and the influence of the treatment protocol and tumour stage on outcome.

\section{METHODS}

Cats treated with RT for sinonasal carcinoma were retrospectively reviewed. Cases that had incomplete radiation protocols or were lost to follow up within two months of protocol completion were excluded. Staging was performed according to the modified Adams' classification system for canine nasal tumours. Three different protocols were used: daily-fractionated (sixteen fractions of 3Gy); cyclic ('quad-shot') palliative (two fractions of 4Gy per day for two consecutive days, repeated every three to four weeks for a total of twelve fractions); palliative (four fractions of 8 or 9 Gy delivered once weekly). Disease free interval (DFI) and median survival time (MST) were calculated using Kaplan-Meier method.

\section{RESULTS}

Sixteen cases were included. The median age at presentation was 13 years (7-14). Half of the cases were classified as Adams' stage 3. Three cats were alive at the time of follow-up. Of the remaining cases, the DFI was $472 \mathrm{~d}$ (77-1428) and the MST was 568d (183-1888). Stage and radiation protocol were not associated with DFI or MST.

\section{STATEMENT (CONCLUSIONS)}

This study population showed improved survival compared to previously reported data, although the overall survival was not associated with tumour stage or the radiation protocol used. Larger studies are necessary to establish a prognostic staging scheme and to evaluate the optimum radiotherapy protocol for feline sinonasal carcinomas.

\section{The toxicity profile of zoledronic acid in dogs - a single centre retrospective study}

\section{David Brewer, Nicholas Bacon, Michael Macfarlane}

Fitzpatrick Referrals Oncology and Soft Tissue, Guildford, United Kingdom

\section{OBJECTIVES}

Intravenous zoledronic acid can be used to treat both bone pain and hypercalcaemia in dogs. There are no reports of its toxicity profile in a large number of canine patients. The primary aim was to report the toxicities in dogs receiving zoledronic acid. A secondary aim was to report the survival time of dogs with primary and secondary bone cancer.

\section{METHODS}

A referral hospital records were retrospectively reviewed Dogs that received at least one dose of zoledronic acid were included if a serum chemistry profile was available within four weeks of the first dose and subsequent administrations. The diagnosis, indication for treatment, and adverse events were documented.

\section{RESULTS}

Ninety-five dogs met the inclusion criteria. Thirty-one received multiple administrations, (range 2-7) of zoledronic acid for a total of 166 administrations, the dose range was $0.13 \mathrm{mg} / \mathrm{kg}-0.32 \mathrm{mg} / \mathrm{kg}, 4-6$ weeks apart Thirteen adverse events were recorded in nine dogs ( $8 \%)$; azotaemia ( $n=8)$, vomiting $(n=2)$, pancreatitis $(n=1)$ cutaneous ulceration $(n=1)$ and diarrhoea $(n=1)$. Zoledronic acid related azotaemia could not be confirmed in any case. Regression analysis demonstrated the change in creatinine was not related to the total dose received $(P=0.51)$. Five dogs $(16 \%)$ changed IRIS stage, the total dose of zoledronic acid received compared to the dogs which did not change IRIS stage was not statistically different $(P=0.93)$. Seventy-one dogs with bone cancer had a median survival time from treatment to death of 110 days, (range $1-589$ days).

\section{STATEMENT (CONCLUSIONS)}

Zoledronic acid appears well tolerated in dogs. A small number developed azotaemia which was not associated with cumulative dose. 